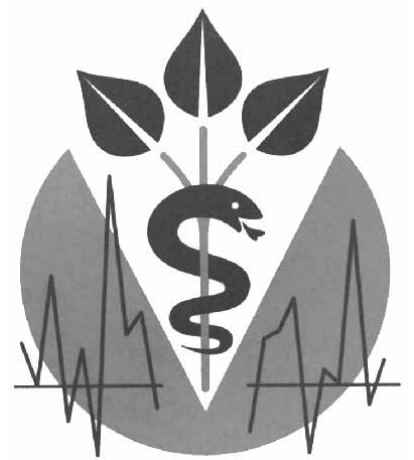

Karl und Veronica Carstens-Stiftung im Stifterverband für die Deutsche Wissenschaft Barkhovenallee 1 45239 Essen

Tel.: 02 01/84 01-111

Fax: 02 01/84 01-302

e-mail: m.fruehwald@stifterverband.de

Neue Veröffentlichungen in der Buchreihe 'edition forschung'

Simon Baumgartner

Der Heidelberger Umweltfragebogen

KVC Verlag, Essen, 1999

DM 32,-

Unsere Umwelt wird zunehmend durch schädliche Chemikalien, Schwermetalle, Gase und andere Luftverunreinigungen belastet. Die damit verbundenen negativen Folgen für die Pflanzen- und Tierwelt sowie die klimatischen und atmosphärischen Veränderungen der Erde sind mittlerweile unübersehbar.

Auch der menschliche Organismus ist von dieser Entwicklung betroffen. Neben einer teilweise exponentiellen Zunahme bestimmter Krankheiten wie z. B. diverser Allergien sind hormonelle, immunologische, metabolische und psychosomatische Störungen zu beobachten.

Um weiteren möglichen Zusammenhängen zwischen Umweltbelastungen und Erkrankungen bzw. Beschwerden, besonders Fertilitätsstörungen, nachgehen $\mathrm{zu}$ können, wurde von der Abteilung für Gynäkologische Endokrinologie und Fertilitätsstörungen der Universitätsfrauenklinik Heidelberg ein umfassender Fragebogen erstellt. Der sogenannte «Heidelberger umfassende Fragebogen zur Umwelt des Patienten» (HUFU) wird in der vorliegenden Arbeit von Simon Baumgartner einer kritischen Analyse unterzogen.

Gesellschaftsmitteilungen - Society Bulletins

Forsch Komplementärmed 1999;6:232

Petra Hampel

Innere Medizin und Naturheilkunde

Die Auseinandersetzung in den Jahren 1882-1933

KVC Verlag, Essen, 1999

DM 32,-

«Unter allen Umständen ist doch die Sorgfalt der Krankenbehandlung die notwendige Eigenschaft eines Menschen, der ein guter Arzt sein will ... und nicht wenige Naturheilkundige sind doch eben Ärzte; sie haben bei uns gelernt. Und das wird immer der gemeinsame Boden sein, auf dem wir uns finden können und finden werden.» (v. Krehl 1935)

Petra Hampel beschreibt in ihrer Arbeit die Auseinandersetzung zwischen den Vertretern der Inneren Medizin und der Naturheilkunde von 1882 bis 1933 anhand der internen therapeutischen Prinzipien bei Diabetes mellitus, Herzinsuffizienz und Tuberkulose. Dabei werden trotz bestehender Differenzen gemeinsame Therapieansätze zwischen beiden «Lagern» sichtbar, die - auch auf die heutige Zeit übertragen - Chancen für eine fruchtbare Zusammenarbeit erkennen lassen.

\section{Jahrbuch der Carstens-Stiftung}

Jahrbuch 5 (1998)

H. Albrecht/M. Frühwald (Hrsg.)

KVC Verlag, Essen, 1999

\section{Inhaltsverzeichnis}

\section{Förderschwerpunkte}

S. Kruse, K. Naske, M. Ulrich und M. Dorcsi: Modellprojekt «Homöopathie in der Pädiatrie»

M. Lacour und R. Huber:

Die Ambulanz für Naturheilverfahren und Umweltmedizin an der Universitätsklinik Freiburg - Zwischenbericht Februar 1998 bis September 1998

\section{Projektförderungen}

U. Albrecht, R. Lüdtke und M. Bühring: Zum Zusammenhang einer chronischen Tonsillitis mit Verquellungen von Bindegewebszonen und einer Brachialgia paraesthetica nocturna

U. Albrecht, R. Lüdtke und M. Bühring:

Offene randomisierte Studie zur Schröpfbehandlung der Brachialgia paraesthetica nocturna

G. Herberth und U. Pison:

Homöopathische Arzneimittel in zellbiologischen Systemen

R. Lüdtke und J. Wilkens:

Klinische Wirksamkeitsstudien zu Arnica in homöopathischen Zubereitungen

Ch. M. Schempp, B. Winghofer, R. Lüdtke, J. C. Simon und E. Schöpf:

Photosensibilisierende und immunmodulierende Eigenschaften von Johanniskrautcreme und Johanniskrautöl

H. Treugut, C. Görner, R. Lüdtke,

P. Schmid und R. Füss:

Neue Aspekte der Kirlian-Fotografie - Reliabilität der Energetischen Terminalpunktdiagnose (ETD) nach Mandel bei klinisch Kranken

H. Treugut， C. Görner， R. Lüdtke und V. Burghardt:

Energetische Meridianmessung mit Prognos- ${ }^{\circledR}-$ Reliabilität

C. Witt, R. Lüdtke, K. Weber, M. Chigne, R. Baur und S. N. Willich:

Die Studie «Verlaufsbeobachtung bei $\mathrm{Pa}$ tienten in der homöopathischen Arztpraxis» - Ein Zwischenbericht

\section{KARGER}

Fax +497614520714

E-mail kargergmbh@aol.com

www.karger.com (c) 1999 S. Karger GmbH, Freiburg

Accessible online at:

http://BioMedNet.com/karger 


\section{Promotionsstipendien}

J. Alex, D. Dieterle, R. Lüdtke, P. C. Endler und H. Moeller:

Die Wirkung homöopathisch potenzierter Thyroxinlösungen auf die Metamorphosegeschwindigkeit des Grasfrosches (Rana temporaria)
J. Hennig, H. P. Ogal, H. Brockmeyer, P. Netter und G. Hempelmann:

Randomisierte verblindete Untersuchung zum Einfluss der Ohrakupunktur bei einer psychischen Belastungssituation («Öffentliches Sprechen»)

\section{Dokumentation}

S. Schober und B. Neundörfer:

Klinische Studien zur Wirksamkeit und Verträglichkeit eines Teeauszuges aus Ruta graveolens bei Sklerose

\section{Schweizerische Jahrestagung für Phytotherapie 18. November 1999 in Baden Phytotherapie bei Beschwerden im Gastrointestinaltrakt}

Programm

$\begin{array}{lll}\text { Zeit } & \text { Thema } & \text { Referent } \\ 09.10 & \text { Begrüssung } & \text { Prof. Dr. B. Meier, Präsident SMGP }\end{array}$

09.15-09.45 Dyspeptische Beschwerden und Phytotherapie - eine Übersicht

09.45-10.15 Dokumentiert für Wissenschaft und Praxis: die Artischocke

10.15-10.30 Diskussion

$10.30-11.00$

$11.00-11.30$

$11.30-12.00$

$12.00-12.15$

$12.15-12.30$

$12.30-14.00$

$14.00-14.30$

$14.30-15.00$

$15.00-15.15$

$14.00-15.15$

$15.15-15.45$

$15.45-16.15$

$16.15-16.45$

$16.45-17.00$

17.15

Anmeldungen an:

\section{Phytotherapie versus Metoclopramid}

Wirksamkeitsnachweis von Phytotherapeutika bei entzündlichen Darmerkrankungen am Beispiel von Boswellia serrata

Diskussion

Verleihung des A. Vogel-Preises 1999

Mittagspause, Ausstellung

Anthrachinondrogen: Bilanz einer Gruppenrevision

Anthrachinonlaxanzien: Nutzen-/Risikopotential

Diskussion

Praxis-Workshop: Erfahrungen mit Magentees bei dyspeptischen Beschwerden

Kaffeepause mit Ausstellung

Neuere Entwicklungen in der Pharmakologie pflanzlicher Gerbstoffe

Was kann die Phytotherapie von der Naturheilkunde lernen? Beispiel: Gastrointestinaltrakt

Schlussdiskussion, Schlusswort

Generalversammlung SMGP
SMGP Schweizerische Medizinische Gesellschaft für Phytotherapie, Kurssekretariat
Prof. Dr. R. Saller, Abteilung für Naturheilkunde, Departement innere Medizin, Universitätsspital Zürich

Prof. Dr. R. Gebhardt, Institut für Biochemie, Leipzig

Dr. med. M.-B. Walle, Mandelbachtal

Dr. med. H. Gerhardt, Colitis-Crohn Ambulanz,

Klinikum Mannheim der Universität Mannheim

Dr. J. Luginbühl, Bioforce AG, Roggwil

Dr. K. Mathys, Interkantonale Kontrollstelle für Heilmittel, Bern

Prof. Dr. med. S. Müller-Lisner, Parkklinik Weisse, Berlin

Prof. Dr. R. Saller und Mitglieder der SMGP

Prof. Dr. O. Sticher, Departement Pharmazie, ETH Zürich

Prof. Dr. M. Bühring, Klinik für Naturheilkunde und Allgemeinmedizin, Krankenhaus Moabit, Berlin

Prof. Dr. B. Meier

Keltenstr. 40

CH-8044 Zürich

Tel./Fax +41 125218 79, E-mail sekretariat-smgp@swissonline.ch

www.smgp.ch 